\title{
The need for standardization of nuclear cardiology reporting and data system (NCAD- RADS): Learning from coronary artery disease (CAD), breast imaging (BI), liver imaging (LI), and prostate imaging (PI) RADS
}

\author{
Majid Assadi, MD, FASNC, ${ }^{\text {E }}$ Erik Velez, MD, ${ }^{\mathrm{b}}$ Mohammad Hosein Najafi, $M D,{ }^{c}$ \\ and Ali Gholamrezanezhad, MD, FEBNM, DABR ${ }^{\mathrm{b}}$ \\ a The Persian Gulf Nuclear Medicine Research Center, Bushehr University of Medical Sciences, \\ Bushehr, Iran \\ b Department of Diagnostic Radiology, Keck School of Medicine, University of Southern \\ California (USC), Los Angeles, CA \\ c Department of Cardiology, Tehran Medical Unit, Azad University, Tehran, Iran
}

Received Jul 18, 2018; accepted Sep 19, 2018

doi: $10.1007 / \mathrm{s} 12350-018-01473-2$

Newer structured reporting manners, the reporting and data system (RADS), have made vast steps in improving standardized and structured reporting, allowing better communication between radiologists and referring providers. This has been implemented in several fields: breast (BI-RADS), lung (Lung-RADS), liver (LI-RADS), thyroid (TI-RADS), prostate (PIRADS), and in cardiovascular radiology (CAD-RADS). The field of nuclear cardiology began its efforts of standardization years ago; however, a widespread standardized reporting structure has not yet been adopted. Such an approach in nuclear cardiology, the nuclear cardiology reporting and data system (NCAD-RADS), will assist radiologists and treating clinicians in conveying and understanding reports and determining the appropriate next steps in management. By linking explicit findings to defined recommendations, patients will receive more consistent and appropriate care.

Key Words: Nuclear cardiology $\cdot$ SPECT $\cdot$ scintigraphy $\cdot$ myocardial perfusion $\cdot$ imaging reporting and data systems $\cdot$ standardization $\cdot$ decision making $\cdot$ report $\cdot$ dictation $\cdot$ diagnosis $•$ interpretation

\begin{tabular}{|llll|}
\hline Abbreviations & LI & Liver imaging \\
NCAD- & Nuclear cardiology reporting and data & PI & Prostate imaging \\
RADS & system & AUC & Appropriate use criteria \\
CAD & Coronary artery disease & & \\
BI & Breast imaging & & \\
\hline
\end{tabular}

Electronic supplementary material The online version of this article (https://doi.org/10.1007/s12350-018-01473-2) contains supplementary material, which is available to authorized users.

The authors of this article have provided a PowerPoint file, available for download at SpringerLink, which summarises the contents of the paper and is free for re-use at meetings and presentations. Search for the article DOI on SpringerLink.com.

Reprint requests: Ali Gholamrezanezhad MD, FEBNM, DABR, Department of Diagnostic Radiology, Keck School of Medicine,
University of Southern California (USC), 1520 San Pablo Street, Suite L1600, Los Angeles, CA, 90033 gholamre@med.usc.edu, a.gholamrezanezhad@yahoo.com

J Nucl Cardiol 2019;26:660-5.

$1071-3581 / \$ 34.00$

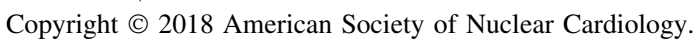




\section{INTRODUCTION}

With the continued digitization of medicine, all medical specialties must strive to adapt while improving patient care. Although this presents unprecedented challenges for the field of radiology, it also allows for unique opportunities. In total, there are three main trends in the digitization of medical imaging: (1) the reporting paradigm, (2) computational and systems biology, and (3) imaging informatics. Radiology must utilize these trends to continue to promote its unique value to patients, payers, and referring providers. ${ }^{1}$

The largest issue facing the reporting paradigm is the widespread adoption of standardized and structured reporting. If achieved, the field of radiology can become the leader in digitized medicine, with searchable report contents and feasible integration with other digitized data. ${ }^{1}$ The radiology community has worked to standardize the communication of results and interpretations by developing numerous guidelines. ${ }^{2}$ Although these guidelines have been very valuable, heterogeneity in the uniformity of the language and structure applied, as well as unjustified final diagnostic conclusions among the different guidelines, has limited their adoption into clinical practice. ${ }^{3}$ Newer structured reporting manners, such as the "*RADS," have made vast steps in improving these issues, allowing for better communication between radiologists and referring providers. ${ }^{4}$ Nevertheless, the continual frequent use of free-text reports for most radiology dictations contradicts the findings of several studies that represent how capturing and analyzing medical data in a structured approach help clinical decision making. ${ }^{5}$ The field of nuclear cardiology began its efforts of standardization years ago., However, a widespread standardized reporting structure has not yet been adopted. Through a coordinated effort, in time, nuclear cardiology imaging reports can accomplish a higher standard of communication with improved comprehensibility in a concise, problem-oriented, and data-driven manner. ${ }^{5,8,9}$

The importance of the "final report" in radiology is highlighted by the fact that it is the main form of communication between radiologists and referring providers. The dangers of non-interactive and nonverbal reporting are the different interpretations referring providers may arrive at through personal bias and the inconsistencies in the communication applied within reports. It is the usual understanding that radiology reports should make available pertinent clinical information and recommendations to ordering clinicians in an understandable format, but only $86 \%$ of radiology reports have been demonstrated to meet this standard. ${ }^{10}$ Moreover, Bosmans et al. ${ }^{11}$ noted a statistically noteworthy difference in not only the length and structure of radiology reports, but in their whole content between practicing radiologists and radiology residents in multiple European nations. Likewise, discordance has been depicted between radiologists and non-radiologists in the degree of diagnostic confidence related to usually applied phrasing in radiology reports. ${ }^{12}$ Many publications over the past decades have suggested different practical and stylistic guidelines. General agreement as to what constitutes a radiology report consists of: an image acquisition protocol, a conscientious interpretation, and a deliberate clinical recommendation. In more comprehensive proposals for the composition of an ideal radiology report, Cramer et al. ${ }^{9}$ and Weiss and Langlotz 13 illustrated the concept of a three-tiered system of structured reporting. The first tier includes the use of common headings, such as "Indication," "Findings," and "Impression." This approach, by far the simplest, is applied by radiologists throughout the world. The second tier expands on the first by adding subheadings, such as organ systems, developing what is known as "itemized reporting",. ${ }^{4}$ The third tier centers around the use of standardized language, and despite being by far the most difficult to implement, is fundamental for the creation of truly structured reporting.

\section{RADIOLOGY LEXICON}

Nowadays, there is remarkable variability in the words applied by radiologists to prepare reports. While this creates flexibility, it is also a cause of confusion. A "lexicon" is described as a vocabulary of words or notions related to their meanings, which if adhered to allow for clearness in communication. ${ }^{14}$ In addition, if the meaning merged in each word or notion can be linked within an established hierarchy, the term itself can be categorized and compared. Notably, this also enables radiology reports to become readily searchable by systems, permitting rapid acquisition of meaningful notions within reports to aid in ongoing analyses and point-of-care questions. ${ }^{1}$

There have been efforts within the radiology community to develop standardized lexicons. ${ }^{15}$ For example, a breast "mass" is described as "a space-occupying lesion seen in two different projections" by the breast imaging reporting and data system (BI-RADS) mammography lexicon ${ }^{16}$ and as uptake with convex outward borders seen on two projections with no interspersed normal uptake on scintimammography by the gamma camera molecular breast imaging lexicon. ${ }^{17,18}$ In spite of these efforts, their applications have been limited, perhaps in part due to the lack of obviously known intrinsic word or notion meaning. In addition, these lexicons have not yet developed an accepted larger hierarchy of meanings or notions. 
Outside the field of radiology, an illuminating lexicon is the ongoing effort by Google called the " The Knowledge Graph'. ${ }^{19}$ In this megaproject, Google is to "organize the world's information and make it universally accessible and useful." Using available free online databases, as well as its own internal cataloging of the Internet as a whole, Google is not only systematically developing a lexicon with the meanings of words, but also with their associating within a hierarchy. On that point, this type of structure would permit precisely the kind of comparison and analysis that could be done with radiology reports, if there is adherence to an agreed-upon lexicon. By extension, when searching for notions or concepts, even if the exact word is not applied in the query, the system retains the capability to return meaningful information. ${ }^{1}$

\section{LEARNING FROM THE * RADS}

The radiology community, with an ongoing coordinated effort by the Radiologic Society of North America (RSNA) titled RadLex, is developing a comprehensive vocabulary for radiology reporting. ${ }^{20}$ This effort is focused on developing a single language that can be used to characterize prominent aspects of an imaging examination (e.g., modality, technique, visual features, anatomy, and pathology). ${ }^{1}$

The early efforts for standardization were made in the late 1980s through the creation of the first version of BI-RADS, established by the American College of Radiology (ACR). ${ }^{21}$ In addition to BI-RADS, standardized reporting has been introduced for several other fields, including: the liver imaging reporting and data system (LI-RADS), ${ }^{22}$ lung CT screening reporting and data system (Lung-RADS), ${ }^{23}$ ACR thyroid imaging reporting and data system (ACR TI-RADS) ${ }^{24}$ and prostate imaging reporting and data system (PIRADS). ${ }^{25}$ In cardiovascular radiology, the coronary artery disease reporting and data system (CAD-RADS) was developed, which can be applied to coronary CT angiography in patients with suspected or known CAD. ${ }^{26}$ These attempts have been widely accepted by third-party payers and the Institute of Medicine as a part of strategies to decrease medical errors that may affect patient outcomes. ${ }^{4}$

Though most radiologists are aware of the *RADS, many may be surprised by the efforts needed for their development. The ACR organized national and international radiology communities, as well as related specialties, in a concerted effort to create the *RADS. ${ }^{22}$ After the first draft proposal, several stages of feedback and iterative revision were needed before the preliminary consensus versions were published. The panels worked on comprehensive lexicons of controlled terminology, uniform reporting, evidence-based management, and appropriate imaging techniques and diagnostic approaches. Afterward, further investigation and updates to these systems were performed, with the inclusion of illustrative atlases and reporting guidelines in efforts to promote and simplify their application. ${ }^{22}$

A main part of the *RADS which has helped achieve the aim of delivering clear and concise reports to referring providers is their associated lexicon, also named "common data elements." The use of a standardized and consistent language style has been demonstrated to promote diagnostic interpretation, improve interobserver consistency, and allow for easier data retrieval and billing while reducing turnaround time and decreasing typographic and dictation errors. ${ }^{9,27}$ Moreover, studies have depicted that consistent terminology can add emphasis to the interpreting radiologist's confidence, considerably influencing a clinician's decision to follow a radiologist's recommendations. ${ }^{28}$ Structured reporting has also been shown to help improve auditing and analysis of data through the application of distinguishable and consistent terminology, which has opened up new and more simplified avenues for research and quality improvement. ${ }^{9}$ As D'Orsi and Kopans mentioned more than 20 years ago, without standardized terms to report the important characters, there is no ways of training or acquiring objective data to progress our specificity. ${ }^{29}$

There must be a concise and systematic description of findings in language familiar for both clinician and radiologist to result in consistent recommendations. Furthermore, standardized reporting has assisted in the understanding of the potential medicolegal consequences of the language applied in radiology reports and may offer potential protections for practicing radiologists. $^{28}$

\section{FUTURE OF RADIOLOGY REPORTING}

As radiologists adapt structured reporting, with a standardized linked lexicon, several opportunities will become possible. Once the concept of structured reporting becomes more familiar, there will be incremental national, and perhaps international, standards for reporting templates. These standards will permit for rapid acquisition and comparison of data for research. The big data pooled from many sources may help in developing a large-scale platform for radiology disease analysis. ${ }^{1}$

Once structured reporting and lexicons become standardized, the next generation of reporting systems will be created. One promising reporting system being developed is the "Multimedia Structured Reporting," presently being pioneered by David J. Vining, MD, of the Image Processing and Visualization Laboratory at 
The University of Texas MD Anderson Cancer Center in Houston. ${ }^{30}$ His product (ViSion) permits multimedia to be merged into the report itself, seamlessly integrating information, such as user-obtained measurements, directly into reports without requiring dictation. ${ }^{1}$

As our communication essentials and expectations alter with new technologies, the medical information that we want to communicate will change as well. Between clinical, imaging, laboratory, and genetic information, referring physicians in the future will face more data than can be handled by one person. Effective incorporation of these varied types of information using "computational biology" or "systems biology" will be essential to distinguish risk and implement of a treatment plan, including suitable imaging in which the role of radiologists will be crucial. ${ }^{31}$ In addition, with the increasing contributions of non-physician providers in the clinical care team, information incorporation and patient-centered imaging approaches will be critical for patient care quality and are best made available by radiologists. ${ }^{1}$

\section{STEP TOWARD A COMMON LEXICON IN NUCLEAR CARDIOLOGY}

Nuclear cardiology use is rapidly growing, and multiple investigations have shown its value in promoting patient care. Nonetheless, nuclear cardiology providers range across the cardiology, radiology and nuclear medicine specialties and each has developed their own styles. In daily practice, these discrepancies are especially evident in two fields: the management of referrals for nuclear cardiology tests and the communication of results. The former gets more attention with constant discussions of proper use criteria and indications for tests by the American Society of Nuclear Cardiology (ASNC) and other international organizations. ${ }^{32}$ In contrast, the communication of the findings has been given significantly less attention. It is therefore imperative that more work is done to create structure and standardization for nuclear cardiology examination reports. ${ }^{33}$

In the era of cardiovascular radiology, the pertinent societies developed CAD-RADS, with the goal of standardizing coronary artery disease severity while incorporating clinical approach recommendations after coronary computed tomography angiography (CTA). ${ }^{26,33,34}$ Standardized reporting is a main quality element of care within cardiovascular imaging. ${ }^{34,35}$ Presently, all cardiovascular radiology modalities have documents for standardized reporting, which include integral parts for image interpretation (e.g., resting heart rate, obstructive stenosis, radiation dose). ${ }^{14,20,36-38}$ Even pretest clinical indications for examinations have been aggregated into multimodality appropriate use criteria(AUC) (i.e. appropriate, maybe appropriate, or rarely appropriate indications). ${ }^{38}$

In the field of nuclear cardiology, there are very limited documentation and recommendations guidelines on reporting imaging examinations. The ASNC published a report to provide imaging guidelines for practitioners who are qualified to practice nuclear cardiology, which was an update of a prior image reporting guideline. ${ }^{7,39}$ In addition, a European position statement - a joint paper by the European Association of Nuclear Medicine (EANM) and the European Association of Cardiovascular Imaging (EACVI) - was developed in an effort to standardize nuclear cardiology reports. ${ }^{6}$ While these efforts have laid the foundation for standardized nuclear cardiology reporting, an internationally standardized reporting structure and lexicon has yet to be developed and adopted on a large scale. Furthermore, due to the remarkable developments in nuclear cardiology, including different modality and hybrid systems, the need for structured reporting is more crucial than ever. ${ }^{6,19,20}$

The development of a common lexicon linking report findings to actionable recommendations will facilitate reporting structure and communication to end users. Such an approach in nuclear cardiology, the nuclear cardiology reporting and data system (NCADRADS), will assist radiologists and treating clinicians in conveying and understanding reports and determining the appropriate next steps in management. Linking explicit diagnoses to defined recommendations for downstream actions are likely to develop consistency in patient care across the spectrum of care providers with different expertise.

It should be mentioned that such protocol could be achievable by collaborative efforts of nuclear medicine, cardiology, and radiology societies with an endeavor to take the best ideas from all stakeholders from national and international communities, with the main goal towards improving patient care. The consideration of different parties in the creation process promoted consensus and facilitated acceptance of the first reporting and data system, BI-RADS, and we are certain this collaborative effort will bear the same fruit.

In conclusion, the advantages of creating a welldefined reporting format and common lexicon in the field of nuclear cardiology, nuclear cardiology reporting and data system (NCAD-RADS), have vast implications: creating an infrastructure of consistent expression, improving communication, and establishing a format for clear, best practice, patient management guidelines. Additionally, this will allow for increased consistency of information, facilitating the acquisition and comparison of research data. The widespread adoption of 
standardized guidelines may facilitate uniformity in patient care across a spectrum of providers with differing expertise and assist in auditing and education.

\section{Disclosure}

None.

\section{References}

1. Li KC, Marcovici P, Phelps A, et al. Digitization of medicine: How radiology can take advantage of the digital revolution. Acad Radiol. 2013;20:1479-94.

2. An C, Rakhmonova G, Choi JY, Kim MJ. Liver imaging reporting and data system (LI-RADS) version 2014: Understanding and application of the diagnostic algorithm. Clin Mol Hepatol. 2016;22:296-307.

3. Davenport MS, Khalatbari S, Liu PS, et al. Repeatability of diagnostic features and scoring systems for hepatocellular carcinoma by using MR imaging. Radiology. 2014;272:132-42.

4. Gholamrezanezhad A, Kessler M, Hayeri SM. The need for standardization of musculoskeletal practice reporting: Learning from ACR BI-RADS, liver imaging-reporting and data system, and prostate imaging-reporting and data system. J Am Coll Radiol. 2017;14:1585-7.

5. Buntin MB, Jain SH, Blumenthal D. Health information technology: Laying the infrastructure for national health reform. Health Aff (Millwood). 2010;29:1214-9.

6. Tragardh E, Hesse B, Knuuti J, et al. Reporting nuclear cardiology: A joint position paper by the European Association of Nuclear Medicine (EANM) and the European Association of Cardiovascular Imaging (EACVI). Eur Heart J Cardiovasc Imaging. 2015;16:272-9.

7. Tilkemeier PL, Cooke CD, Grossman GB, McCallister BD, Ward RP. Standardized reporting of radionuclide myocardial perfusion and function. J Nucl Cardiol. 2009;16:650.

8. Bosmans JM, Van Goethem JW, De Schepper AM. Structure and content of the radiological report: An audit of 94 reports from a university education center. JBR-BTR. 2004;87:260-4.

9. Cramer JA, Eisenmenger LB, Pierson NS, Dhatt HS, Heilbrun ME. Structured and templated reporting: An overview. Appl Radiol. 2014;43:18-21.

10. Lee R, Cohen MD, Jennings GS. A new method of evaluating the quality of radiology reports. Acad Radiol. 2006;13:241-8.

11. Bosmans JM, Weyler JJ, Parizel PM. Structure and content of radiology reports, a quantitative and qualitative study in eight medical centers. Eur J Radiol. 2009;72:354-8.

12. Khorasani R, Bates DW, Teeger S, Rothschild JM, Adams DF, Seltzer SE. Is terminology used effectively to convey diagnostic certainty in radiology reports? Acad Radiol. 2003;10:685-8.

13. Weiss DL, Langlotz CP. Structured reporting: Patient care enhancement or productivity nightmare? Radiology. 2008;249:739-47.

14. Leipsic J, Abbara S, Achenbach S, et al. SCCT guidelines for the interpretation and reporting of coronary $\mathrm{CT}$ angiography: A report of the Society of Cardiovascular Computed Tomography Guidelines Committee. J Cardiovasc Comput Tomogr. 2014;8:342-58.

15. Hansell DM, Bankier AA, MacMahon H, McLoud TC, Muller NL, Remy J. Fleischner society: Glossary of terms for thoracic imaging. Radiology. 2008;246:697-722.
16. Rao AA, Feneis J, Lalonde C, Ojeda-Fournier H. A pictorial review of changes in the BI-RADS Fifth Edition. Radiographics. 2016;36:623-39.

17. Conners AL, Hruska CB, Tortorelli CL, et al. Lexicon for standardized interpretation of gamma camera molecular breast imaging: observer agreement and diagnostic accuracy. Eur J Nucl Med Mol Imaging. 2012;39:971-82.

18. Conners AL, Maxwell RW, Tortorelli CL, et al. Gamma camera breast imaging lexicon. AJR Am J Roentgenol. 2012;199:W76774.

19. Flotats A, Knuuti J, Gutberlet M, et al. Hybrid cardiac imaging: SPECT/CT and PET/CT. A joint position statement by the European Association of Nuclear Medicine (EANM), the European Society of Cardiac Radiology (ESCR) and the European Council of Nuclear Cardiology (ECNC). Eur J Nucl Med Mol Imaging. 2011;38:201-12.

20. Tilkemeier PL, Cooke CD, Ficaro EP, et al. American Society of Nuclear Cardiology information statement: Standardized reporting matrix for radionuclide myocardial perfusion imaging. J Nucl Cardiol. 2006;13:e157-71.

21. Burnside ES, Sickles EA, Bassett LW, et al. The ACR BI-RADS experience: Learning from history. J Am Coll Radiol. 2009;6:85160.

22. Mitchell DG, Bruix J, Sherman M, Sirlin CB. LI-RADS (Liver Imaging Reporting and Data System): Summary, discussion, and consensus of the LI-RADS Management Working Group and future directions. Hepatology. 2015;61:1056-65.

23. Kazerooni EA, Armstrong MR, Amorosa JK, et al. ACR CT accreditation program and the lung cancer screening program designation. J Am Coll Radiol. 2016;13:R30-4.

24. Tessler FN, Middleton WD, Grant EG, et al. ACR Thyroid Imaging, Reporting and Data System (TI-RADS): White Paper of the ACR TI-RADS Committee. J Am Coll Radiol. 2017;14:58795.

25. Rosenkrantz AB, Kim S, Lim RP, et al. Prostate cancer localization using multiparametric MR imaging: Comparison of Prostate Imaging Reporting and Data System (PI-RADS) and Likert scales. Radiology. 2013;269:482-92.

26. Cury RC, Abbara S, Achenbach S, et al. CAD-RADS(TM) Coronary Artery Disease-Reporting and Data System. An expert consensus document of the Society of Cardiovascular Computed Tomography (SCCT), the American College of Radiology (ACR) and the North American Society for Cardiovascular Imaging (NASCI). Endorsed by the American College of Cardiology. J Cardiovasc Comput Tomogr. 2016;10:269-81.

27. Liu D, Zucherman M, Tulloss WB Jr. Six characteristics of effective structured reporting and the inevitable integration with speech recognition. J Digit Imaging. 2006;19:98-104.

28. Srinivasa Babu A, Brooks ML. The malpractice liability of radiology reports: Minimizing the risk. Radiographics : a review publication of the Radiological Society of North America, Inc. 2015;35:547-554.

29. D'Orsi CJ, Kopans DB. The American College of Radiology's mammography lexicon: Barking up the only tree. AJR Am J Roentgenol. 1994;162:595.

30. A. M. (2012) Sketching a new reality: What will the radiology report of the future look like? In: ACR News. http://www.acr.org/ News-Publications/News/News-Articles/2012/ACR-Bulletin/2012 03-Rad-Report-of-Future.

31. Ghasemi M, Nabipour I, Omrani A, Alipour Z, Assadi M. Precision medicine and molecular imaging: New targeted approaches toward cancer therapeutic and diagnosis. Am J Nucl Med Mol Imaging. 2016;6:310-27. 
32. Brindis RG, Douglas PS, Hendel RC, et al. ACCF/ASNC appropriateness criteria for single-photon emission computed tomography myocardial perfusion imaging (SPECT MPI): A report of the American College of Cardiology Foundation Quality Strategic Directions Committee Appropriateness Criteria Working Group and the American Society of Nuclear Cardiology endorsed by the American Heart Association. J Am Coll Cardiol. 2005;46:1587-605.

33. Chandrashekhar Y, Min JK, Hecht H, Narula J. CAD-RADS: A giant first step toward a common lexicon? JACC Cardiovasc Imaging. 2016;9:1125-9.

34. Xie JX, Cury RC, Leipsic J, et al. The Coronary Artery DiseaseReporting and Data System (CAD-RADS): Prognostic and clinical implications associated with standardized coronary computed tomography angiography reporting. JACC Cardiovascular imaging. 2018;11:78-89.

35. Douglas P, Iskandrian AE, Krumholz HM, et al. Achieving quality in cardiovascular imaging: Proceedings from the American College of Cardiology-Duke University Medical Center Think Tank on Quality in Cardiovascular Imaging. J Am Coll Cardiol. 2006;48:2141-51.

36. Gardin JM, Adams DB, Douglas PS, et al. Recommendations for a standardized report for adult transthoracic echocardiography: A report from the American Society of Echocardiography's Nomenclature and Standards Committee and Task Force for a
Standardized Echocardiography Report. J Am Soc Echocardiogr. 2002;15:275-90.

37. Schulz-Menger J, Bluemke DA, Bremerich J, et al. Standardized image interpretation and post processing in cardiovascular magnetic resonance: Society for Cardiovascular Magnetic Resonance (SCMR) board of trustees task force on standardized post processing. J Cardiovasc Magn Reson. 2013;15:35.

38. Wolk MJ, Bailey SR, Doherty JU, et al. ACCF/AHA/ASE/ASNC/ HFSA/HRS/SCAI/SCCT/SCMR/STS 2013 multimodality appropriate use criteria for the detection and risk assessment of stable ischemic heart disease: A report of the American College of Cardiology Foundation Appropriate Use Criteria Task Force, American Heart Association, American Society of Echocardiography, American Society of Nuclear Cardiology, Heart Failure Society of America, Heart Rhythm Society, Society for Cardiovascular Angiography and Interventions, Society of Cardiovascular Computed Tomography, Society for Cardiovascular Magnetic Resonance, and Society of Thoracic Surgeons. J Am Coll Cardiol. 2014;63:380-406.

39. Dorbala S, Ananthasubramaniam K, Armstrong IS, et al. Single Photon Emission Computed Tomography (SPECT) Myocardial Perfusion Imaging Guidelines: Instrumentation, acquisition, processing, and interpretation. J Nucl Cardiol. 2018. https://doi.org/ 10.1007/s12350-018-1283-y. 